\title{
GAIT RECOGNITION BASED ON 3D HOLOSCOPIC GAIT ENERGY IMAGE
}

\author{
Hakan Ozen ${ }^{1,2 *}$, Nikolaos V. Boulgouris ${ }^{1}$, and Rafiq Swash ${ }^{1}$ \\ ${ }^{1}$ Brunel University London, United Kingdom \\ ${ }^{2}$ Middle East Technical University
}

\begin{abstract}
We present a novel system for gait recognition based on holoscopic rather than conventional imaging. The system is based on a novel gait template, the Holoscopic Gait Energy Image (HGEI), that is constructed from holoscopic rather than conventional images. Due the holoscopic recording approach, the proposed template captures richer information and the resultant system has increased capabilities. The proposed system is compared to the conventional approach for gait recognition using a newly recorded database and is experimentally shown to yield favourable results.
\end{abstract}

Index Terms - Gait, recognition, holoscopic, imaging, 3D, silhouette.

\section{INTRODUCTION}

Although the field of gait recognition [1] has seen significant advances in the past few years, there are still several challenges that have to be met in order to enable the reliable operation of gait recognition technology outside the laboratory. Such challenges have to do with the ability to operate effectively, regardless of the age, emotional state, and clothing of the observed subjects, as well as to deliver satisfactory recognition performance in outdoor areas, where occlusions and complicated backgrounds are to be expected.

An imaging methodology that can contribute to meeting some of the current challenges in gait recognition and enable the widespread use of gait as a reliable biometric is the development of $3 \mathrm{D}$ holoscopic cameras, where a $3 \mathrm{D}$ object is imaged through an array of micro lenses [2]. Each micro lens in the lens array captures a 2D elemental image of the object from a unique perspective that depends on the position of the lens in the lens array. The availability of multiple images, captured from slightly different viewpoints, enables the subsequent reconstruction of 3D objects. This approach, inspired from the vision system of the fly, is fundamentally different from methods that deploy a simple depth map [3].

The general framework of automatic gait recognition consists of subject detection, feature extraction, and classification. There are mainly two kinds of gait features, namely

${ }^{*}$ This work was largely completed while H. Ozen was with Brunel University London.

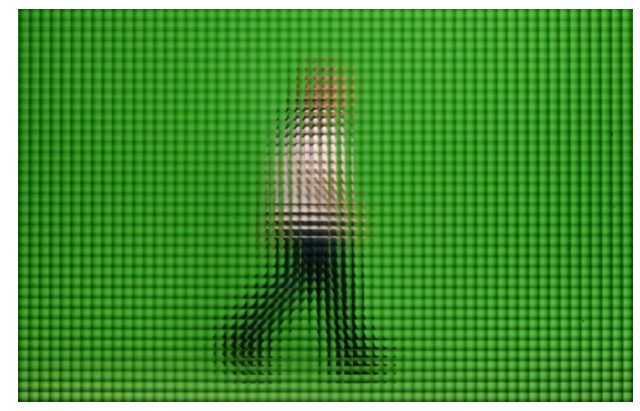

Fig. 1. Raw holoscopic image representation.

model-based and model-free features. Model-free features are normally acquired from binary silhouettes of the walking subject. Using walking silhouettes, distinctive gait patterns can be extracted $[4,5]$ and used as gait signatures. The most popular model-free gait representation is the Gait Energy Image (GEI) [4], which averages silhouettes in a gait cycle. Although the averaging operation apparently discards gait dynamics, the GEI is a very effective gait representation.

In this paper, we focus on a model-free gait recognition approach, which relies on holoscopic rather than conventional silhouettes. Gait information is captured using a simple lens array that is attached on a conventional camera and does not impose increased equipment requirements on the architecture of the system. Using the holoscopic silhouettes, we construct a holoscopic template, the Holoscopic Gait Energy Image (HGEI), which is subsequently used for recognition. Due the holoscopic recording principles, our proposed holoscopic template captures richer information than the conventional GEI. Furthermore, the new system has increased human detection and background subtraction capabilities, which are expected to be particularly useful in cases of complex scenes captured in crowded environments where occlusion or complicated backgrounds are common.

The paper is organized as follows. The holoscopic representation is presented in Section 2. The construction of the holoscopic templates is described in Section 3, while the classification methodology is presented in Section 4. Experimental evaluation is presented in Section 5 and, finally, conclusions are drawn in Section 6. 


\section{HOLOSCOPIC REPRESENTATION}

\begin{tabular}{|l|l|l|l|l|l|l|l|l|}
\hline 1 & 1 & 1 & 2 & 2 & 2 & 3 & 3 & 3 \\
\hline 1 & 1 & 1 & 2 & 2 & 2 & 3 & 3 & 3 \\
\hline 1 & 1 & 1 & 2 & 2 & 2 & 3 & 3 & 3 \\
\hline 4 & 4 & 4 & 5 & 5 & 5 & 6 & 6 & 6 \\
\hline 4 & 4 & 4 & 5 & 5 & 5 & 6 & 6 & 6 \\
\hline 4 & 4 & 4 & 5 & 5 & 5 & 6 & 6 & 6 \\
\hline 7 & 7 & 7 & 8 & 8 & 8 & 9 & 9 & 9 \\
\hline 7 & 7 & 7 & 8 & 8 & 8 & 9 & 9 & 9 \\
\hline 7 & 7 & 7 & 8 & 8 & 8 & 9 & 9 & 9 \\
\hline
\end{tabular}

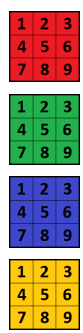

Fig. 2. A holoscopic image (left) and its constituent viewpoint images (right).

Holoscopic image recording is achieved by means of an array of lenses, where every lens records a unique 2D image of the $3 \mathrm{D}$ object. The representation of a holoscopic image is graphically explained in Fig. 2. On the right-hand side of Fig. 2, the constituent unique 2D images, captured from slightly different perspectives, are shown as images of low resolution. These images are the viewpoint images and their number depends on the design of the lens array. On the lefthand side of Fig. 2, the constituent images are re-arranged in a full image representation that resembles a 2D image of full resolution. An example of such an image is shown in Fig. 1.

The detail of a holoscopic representation is shown in Fig. 3(b). The original holoscopic viewpoint representation, shown in Fig. 3 (a), comprises $29 \times 29$ viewpoint images. As the number of viewpoint images increases, their resolutions decrease. However, the deployment of silhouette-based features in our architecture simplifies the operation of the system and makes it less critically dependent on the resolution of the constituent viewpoint images.

\section{HOLOSCOPIC GAIT TEMPLATE CONSTRUCTION}

In order to construct a holoscopic template for recognition purposes, we first extract binary holoscopic silhouettes from the recorded holoscopic image sequences. The holoscopic silhouettes are subsequently aligned in the centre of the frame and they are cropped in order to include only the walking subject. The output of this process is graphically shown in Fig. 4.

Following silhouette extraction, a standard method (e.g., like the one in [6]) is used for partitioning the sequence of silhouettes into gait cycles. Similar to the construction of the conventional GEI, holoscopic silhouettes in a gait cycle are subsequently used for the construction of a Holoscopic Gait

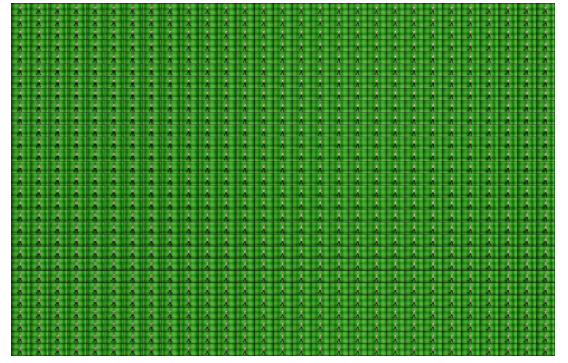

(a)

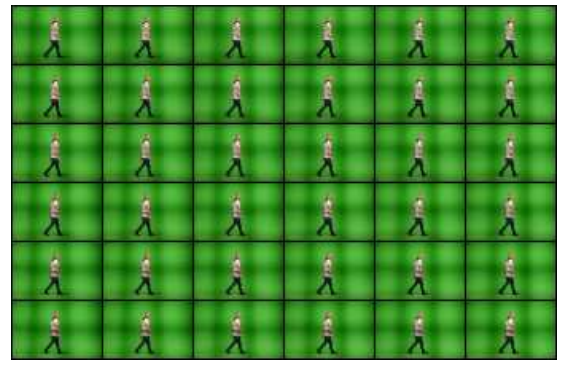

(b)

Fig. 3. Structure of a 3D Holoscopic image, (a) $29 \times 29$ array of viewpoint images, (b) Zoomed $6 \times 6$ part of the array.

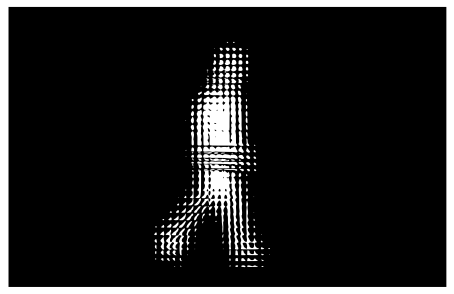

(a)

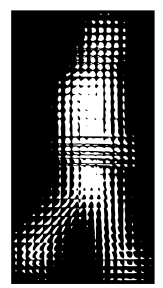

(b)
Fig. 4. Holoscopic silhouette. (a) Original, (b) Centered and cropped.

Energy Image (HGEI) by means of averaging:

$$
\operatorname{HGEI}(x, y)=\frac{1}{T} \sum_{t=1}^{T} h_{t}(x, y),
$$

where $T$ is the number of holoscopic silhouettes in a gait cycle and $h_{t}$ represents the holoscopic silhouette at time $t$. This combination of multiple holoscopic silhouettes into a HGEI template is shown in Figure 5.

A GEI and a HGEI corresponding to the same walking sequence are shown in Fig. 6. Despite being a binary template, the HGEI captures richer information due the its ability to capture elements of the observed subject's 3D structure. This approach is substantially different from that used in conventional GEI, which mainly captures the temporal imprints of human contours and for this reason it would lead to less effective gait representations. It has to be noted that, in this work, we endeavour to assess the inherent gait representation capacity of the holoscopic template and we do not investigate 


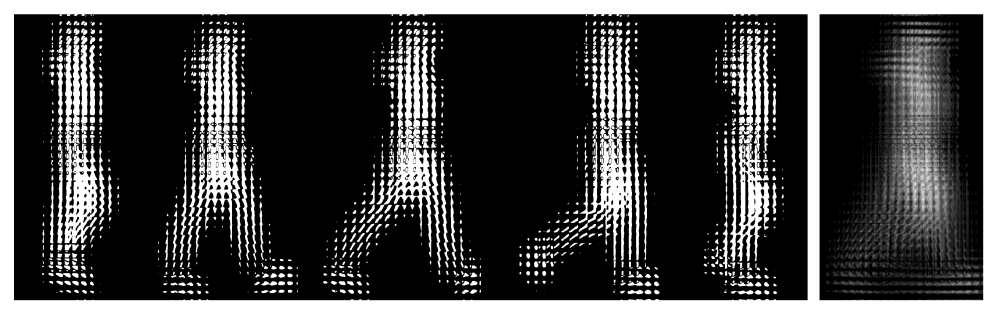

Fig. 5. Construction of Holoscopic GEI (right-most image) using holoscopic silhouettes.

additional feature extracting techniques, such as Linear Discriminant Analysis [7] or Deep Neural Networks [8], which can be applied in conjunction with template-based gait representations.

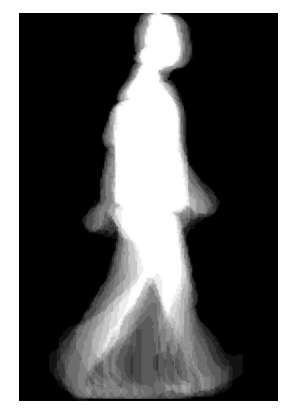

(a)

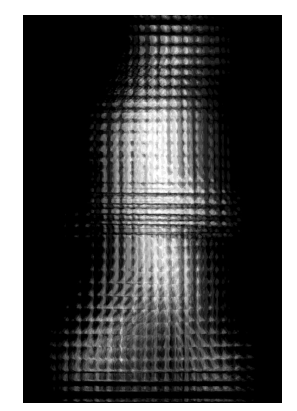

(b)
Fig. 6. (a) Conventional GEI, (b) Proposed holoscopic GEI.

\section{GAIT TEMPLATE CLASSIFICATION}

For classifying the templates constructed as above, we performed template matching by trying minor horizontal displacements to compensate for the fact that direct matching is rendered ineffective in cases where reference and test sequences correspond to different walking conditions. Such displacements have been seen to be useful in the context of gait recognition [9] in situations where carried bags or heavy clothing shift the centre of the template and result in distances that do not reflect the true likeness between the observed subjects. Considering these, for both GEI and HGEI, the distance between the $i$ th reference template $R_{i}, i=1, \ldots, N$ and the $j$ th test template $P_{j}$ is calculated as

$$
D_{i j}\left(\delta_{x}\right)=\sum_{(x, y)}\left(R\left(x+\delta_{x}, y\right)-P(x, y)\right)^{2}
$$

where $\delta_{x}$ is the applied horizontal displacement. For each comparison between reference and test templates, we tried the above distance calculation for displacements $\delta_{x}=-5, \ldots, 5$ and we kept the minimum, i.e., using the the $\delta_{x}$ that yielded the minimum distance $\tilde{D}$. The search for minimum distance along a $1 D$ line is reminiscent of disparity calculation in stereo imaging or displaced frame difference in motion estimation [10]. Due to the fact that only horizontal displacements were considered, the distance calculation based on eq. (2) above remains extremely fast and imposes negligible additional computational load on the recognition process. Once all minimum distances $\tilde{D}_{i j}$ between the $j$ th test template and reference templates $i=1, \ldots, N$ are calculated, the index $i^{*}$ of the recognized subject is determined as

$$
i^{*}=\arg \max _{i} \tilde{D}_{i j}
$$

As we will see in the ensuing section, the HGEI template offers performance advantages in comparison to the conventional approach.

\section{EXPERIMENTAL RESULTS}

For the experimental evaluation of our methodology, we recorded a small detabase of holoscopic gait sequences using seven volunteering subjects. The seven subjects were recorded in three different conditions, i.e., without carrying objects, carrying a bag on their left shoulder, and carrying a bag on their right shoulder. We then compiled a Reference set of gait representations, which includes the subjects walking without carrying objects, as well as two test (Probe) sets in which the subjects were carrying a bag on their left (Probe A) or right shoulder (Probe B).

For each recorded sequence we constructed a conventional Gait Energy Image (GEI) as well as our proposed Holoscopic GEI (HGEI). As expected when using a small number of subjects observed in lab conditions, recognition performance was $100 \%$ for both GEI and HGEI, which did not lead to conclusive results. For this reason, we made the recognition problem harder by using portions of the GEI and HGEI for recognition, i.e., truncated templates from which the upper portion of the full template was left out.

As seen in Fig. 7, the holoscopic approach yields improved results in comparison to the conventional GEI and exhibits very promising performance. Interestingly, the performance gains of the HGEI over the GEI are more pronounced when the part of the template that represents the carried bag is included in the truncated template that is used.

It is important to note that the holoscopic approach endows the proposed scheme not only with the capability for 


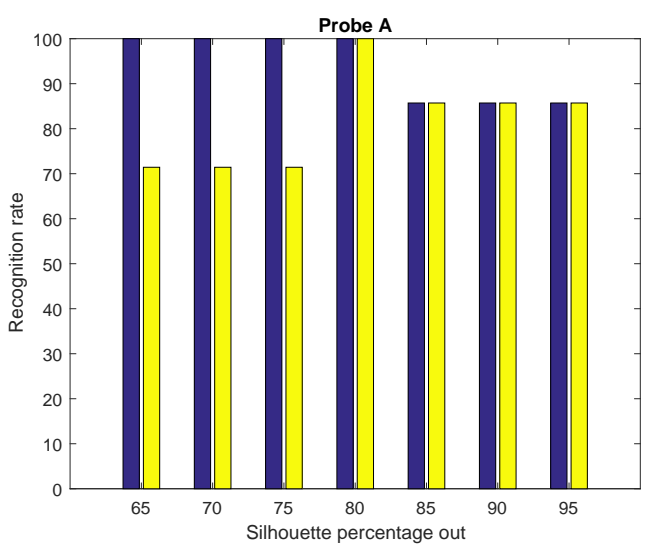

(a)

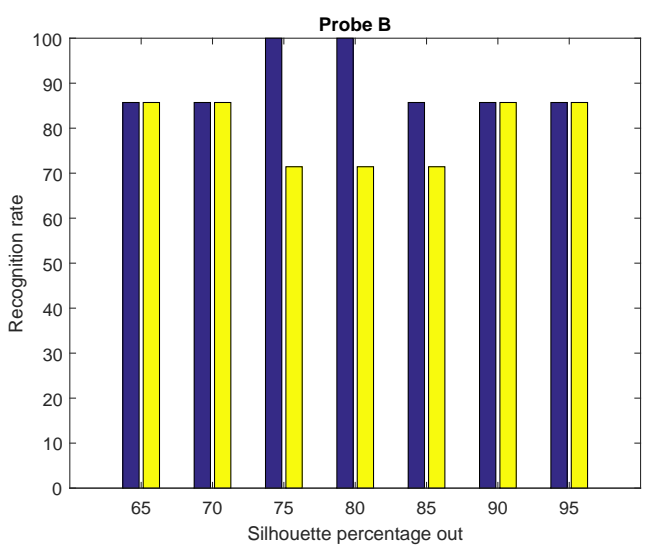

(b)

Fig. 7. Comparison between HGEI and GEI when portions of the templates are left out, (a) Probe A, (b) Probe B. Blue bars represent the HGEI and yellow bars represent the GEI (best viewed in color).

superior recognition performance but also with functionalities that would be very valuable in a real-life recording situations, where the background is not uniform or the scene is crowded and there are occlusions. In such cases, the depth estimation that the holoscopic representation allows will enable the system to perform more accurate extraction of the target subject leading to further performance gains.

\section{CONCLUSION}

We proposed a novel system for gait recognition based on holoscopic rather than conventional imaging. The new system is based on a simple array of lenses that is attached on conventional cameras and, therefore, it does not impose increased equipment requirements. The system is based on a holoscopic template, the Holoscopic Gait Energy Image (HGEI), that is constructed from holoscopic rather than conventional images. Due the holoscopic recording approach, the new system has increased background subtraction capabilities, which are particularly useful in cases of complex scenes captured in crowded environments where occlusion or complicated backgrounds are to be expected. The system is compared to the conventional approach for gait recognition using a newly recorded database and is experimentally shown to produce very favourable results.

\section{REFERENCES}

[1] N.V. Boulgouris, D. Hatzinakos, and K.N. Plataniotis, "Gait recognition: a challenging signal processing technology for biometric identification," IEEE Signal Processing Magazine, vol. 22, pp. 78-90, Nov. 2005.

[2] A. Aggoun, E. Tsekleves, M. R. Swash, D. Zarpalas, P. Daras, P. Nunes, and D. Soares, "Immersive 3D holoscopic video system," IEEE Multimedia, vol. 20, no. 1, pp. 28-37, Jan.-Mar. 2013.

[3] D. Ioannidis, D. Tzovaras, I. G. Damousis, S. Argyropoulos, and K. Moustakas, "Gait recognition using compact feature extraction transforms and depth information," IEEE Trans. Information Forensics and Security, vol. 2, no. 3, pp. 623-630, Sep. 2007.

[4] J. Han and B. Bhanu, "Individual recognition using gait energy image," IEEE Trans. Pattern Anal. Machine Intell., vol. 28, no. 2, pp. 316-322, 2006.

[5] K. Bashir, T. Xiang, and S. Gong, "Gait recognition without subject cooperation," Pattern Recognition Letters, vol. 31, no. 13, pp. 2052-2060, Oct. 2010.

[6] N.V. Boulgouris, K.N. Plataniotis, and D. Hatzinakos, "Gait recognition using dynamic time warping," in Proc. IEEE Int. Symp. Multimedia Signal Processing, Siena, Italy, Sep. 2004, pp. 263-266.

[7] R. O. Duda, P. E. Hart, and D. G. Stork, Pattern Classification, John Wiley \& Sons, Inc., 2001.

[8] Z. Wu, Y. Huang, L. Wang, X. Wang, and T. Tan, “A comprehensive study on cross-view gait based human identification with deep CNNs," IEEE Transactions on Pattern Analysis and Machine Intelligence, vol. 39, no. 2, pp. 209 - 226, Feb. 2017.

[9] X. Huang and N. .V. Boulgouris, "Gait recognition with shifted energy image and structural feature extraction," IEEE Transactions on Image Processing, vol. 21, pp. 2256-2268, 2012.

[10] A. M. Tekalp, Digital Video Processing, Prentice Hall, 2015. 